\title{
Glucose Availability and the Growth Rate of Colonies of Pseudomonas fluorescens
}

\author{
By V. T. RIECK, S. A. PALUMBO* AND L. D. WITTER \\ Department of Food Science, University of Illinois, Urbana, Illinois, 61801, U.S.A.
}

(Received Io April I972; revised I I July 1972)

\begin{abstract}
SUMMAR Y
Colonies of Pseudomonas fluorescens growing on glucose salts agar with low concentrations of glucose ( $0^{\circ} \mathrm{I}$ to $\left.0.0 \mathrm{\%} \%\right)$ exhibit 'transition points' or times at which the initial diameter increase changes to a second and slower linear rate. As the concentration of glucose was lowered, the colonies had slower rates of diameter increase and earlier transition points. Growth of the colonies after the transition point was shown to be limited by the rate of diffusion of glucose and by its availability to the colony. The transition point occurred when the amount of glucose available to the colony by extension (growth) of the colony started to decrease and when there was a minimum value for the total amount of glucose available per viable organism in the colony.
\end{abstract}

\section{INTRODUCTION}

Palumbo, Johnson, Rieck \& Witter (I971), from their own studies and those of others (Pirt, I967; Cooper, Dean \& Hinshelwood, I968), proposed that a linear increase of colony diameter with time represented the steady state growth phase of bacterial colonies. This linear increase of colony diameter with time was observed with conditions of different temperatures (Johnson, Palumbo, Rieck \& Witter, 1970) and with various nutrients (Palumbo et al. 1971).

Under certain conditions, e.g. low concentrations of glucose, colonies of Pseudomonas fluorescens exhibited 'transition points' or times at which the initial linear phase changed to a second and slower linear phase. Pirt (1967) showed that such a transition point also occurred for colonies of Escherichia coli, and obtained similar data for colonies of Klebsiella aerogenes and Streptococcus faecalis. Palumbo et al. (I97I) could not attribute the transition points which they observed to a diauxie effect (Monod, 1942) or to secondary colonies (Lewis, I933; Shah \& Iyer, I96I) since they occurred when the colonies were growing on glucose salts agar which contained a single carbon and a single nitrogen source. The transition point could not be explained by accumulation of toxic metabolic products since this strain of $P$. fluorescens is known not to produce any toxic products when grown in glucose salts broth (Sinclair \& Stokes, 1962). The present study suggested that the transition point occurred when the growth of the colony was limited by the availability of glucose.

* Present address: Meat, Hides and Leather Laboratory, Eastern Marketing and Nutrition Research Division, 600 East Mermaid Lane, Philadelphia, Pennsylvania, 19118, U.S.A. Author to whom reprint requests should be addressed.

Vol. 73, No. 3, was issued 3 January 1973 


\section{METHODS}

General. The organism, media preparation, culture conditions, $\Delta d / \Delta t$ measurements and colony viable organism counts were described by Palumbo et al. (197I).

Inoculation. Glucose salts agar plates (GSA) were inoculated either by the surface plating technique (Palumbo et al. I97I) or by the single colony method (Bates, I929; Shrewberry, I93I).

Glucose analysis. The concentration of glucose was determined by the glucostat method (Worthing Biochemical Corporation, Freehold, New Jersey, U.S.A.).

Coefficient of diffusion. The coefficient of diffusion of glucose through GSA was determined at $30^{\circ} \mathrm{C}$ by the procedures of Lauffer (196I) and Shantz \& Lauffer (1962).

Glucose utilization by colonies. Single colonies of Pseudomonas fluorescens were inoculated at the centre of Petri dishes of GSA. At intervals colony diameters were measured and the colonies removed for viable organism counts. Agar sections adjacent to the colony were removed and analysed for glucose. The agar was first cut tangent to the colony and then two parallel cuts $(0.48 \mathrm{~cm}$ apart) perpendicular to the first were made away from the colony to the edge of the Petri dish; the midpoint of the strip formed was at the point where the first cut was tangent to the colony. The strip was finally cut into squares. Each of these squares was of known dimensions and was analysed for its glucose concentration.

\section{RESULTS}

Establishment of the transition point. One of the first considerations was whether the transition point was a real phenomenon or whether it was a function of how the data were presented. To determine the existence of a transition point or change in rate of growth and the exact nature of the response of a colony to decreases in the glucose concentration in the GSA medium, data from a normal ( $\mathrm{I} \%$ ) glucose and a low $(0 . \mathrm{I} \%$ ) glucose were subjected to analysis of variance and multiple linear regression analysis. On I \% glucose GSA medium, the diameter of colonies of Pseudomonas fluorescens increased linearly with time. The equation which fits the data is: $Y$ (diameter) $=-\mathrm{I} \cdot 37736+0.05457$ (time) \pm 0.43 . However, on $0.1 \%$ glucose GSA medium, colony diameter increase could be either quadratic or two straight lines. The respective equations are $(a)$ quadratic:

$$
Y=-2 \cdot 0557+0 \cdot 08954(\text { time })-0 \cdot 00024\left(\text { time }^{2}\right) \pm 0 \cdot 36 \text {; }
$$

and $(b)$ two straight lines:

and

$$
Y(\mathrm{I})=-\mathrm{I} \cdot 256+0.05602 \text { (time) up to } 82 \mathrm{~h}
$$

$$
Y(2)=-0.8043+0.03136(\text { time }) \text { after } 95 \mathrm{~h} \pm 0.38 .
$$

Growth rate of colonies. The $\Delta d / \Delta t$ of a colony of Pseudomonas fluorescens influenced by nearby colonies was compared with that of a single colony of $P$. fluorescens on an agar plate in Fig. I. Under crowded conditions ( 54 colonies/plate) one colony grew at an initial $\Delta d / \Delta t$ of $0.050 \mathrm{~mm} / \mathrm{h}$. After I02 h the $\Delta d / \Delta t$ decreased to $0.017 \mathrm{~mm} / \mathrm{h}$. However, when only one colony was present on a plate, the average $\Delta d / \Delta t$ of four randomly chosen colonies was $0.059 \mathrm{~mm} / \mathrm{h}$. The single colonies grew at this rate until the experiment was terminated at $469 \mathrm{~h}$. Fig. I also shows that no maximum colony diameter can be assigned to $P$. fluorescens.

Glucose concentrations of $\mathrm{I} \%$ and $0.5 \%$ had little effect on $\Delta d / \Delta t$ and no transition 


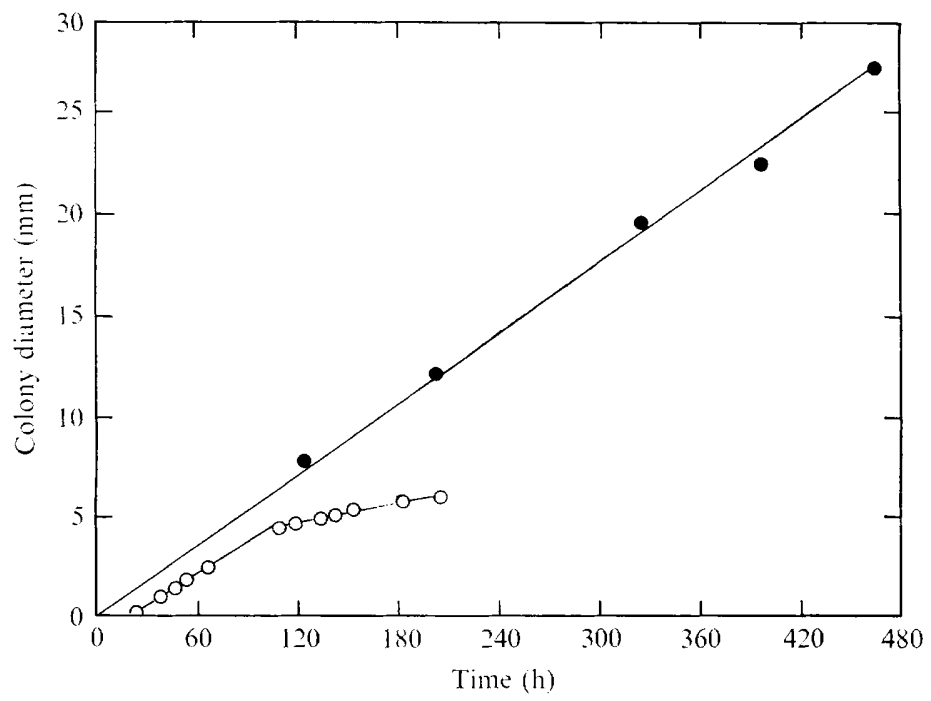

Fig. I. The influence of number of colonies per plate on the $\Delta d / \Delta t$ for Pseudomonas fluorescens at $25^{\circ} \mathrm{C}$. One colony/plate; $\bigcirc, 54$ colonies/plate.

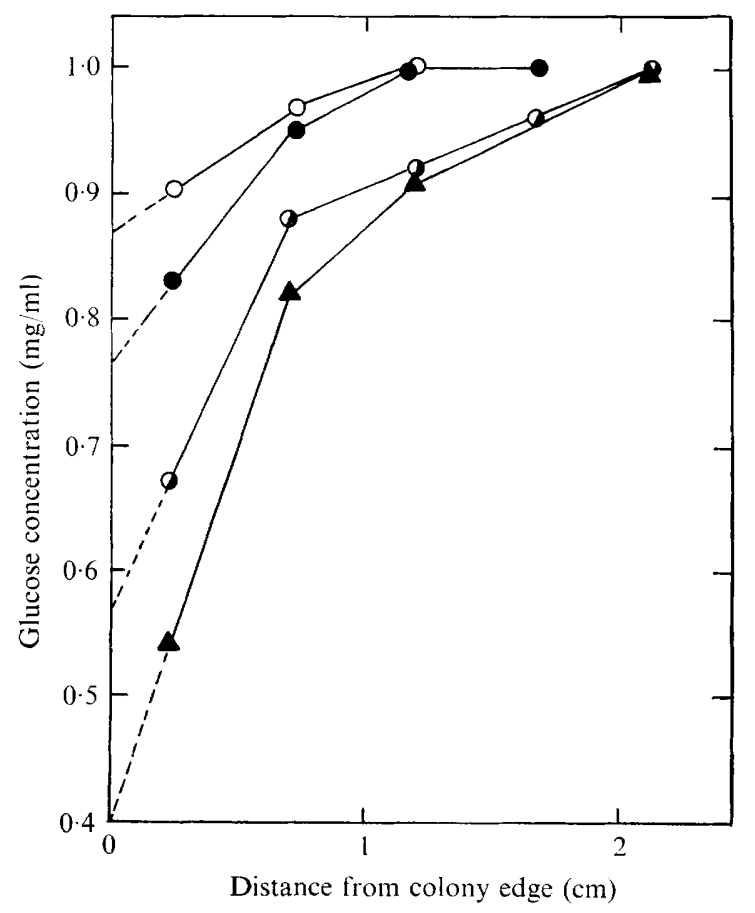

Fig. 2. The glucose concentration at various distances from the edge of Pseudomonas fuorescens colonies growing on GSA with $0.1 \%$ glucose. $0,52 \mathrm{~h} ; \boldsymbol{0}, 83 \mathrm{~h} ; \boldsymbol{\top}, 97 \mathrm{~h} ; \boldsymbol{\Delta}, \mathrm{I} 2 \mathrm{I} \mathrm{h}$. 


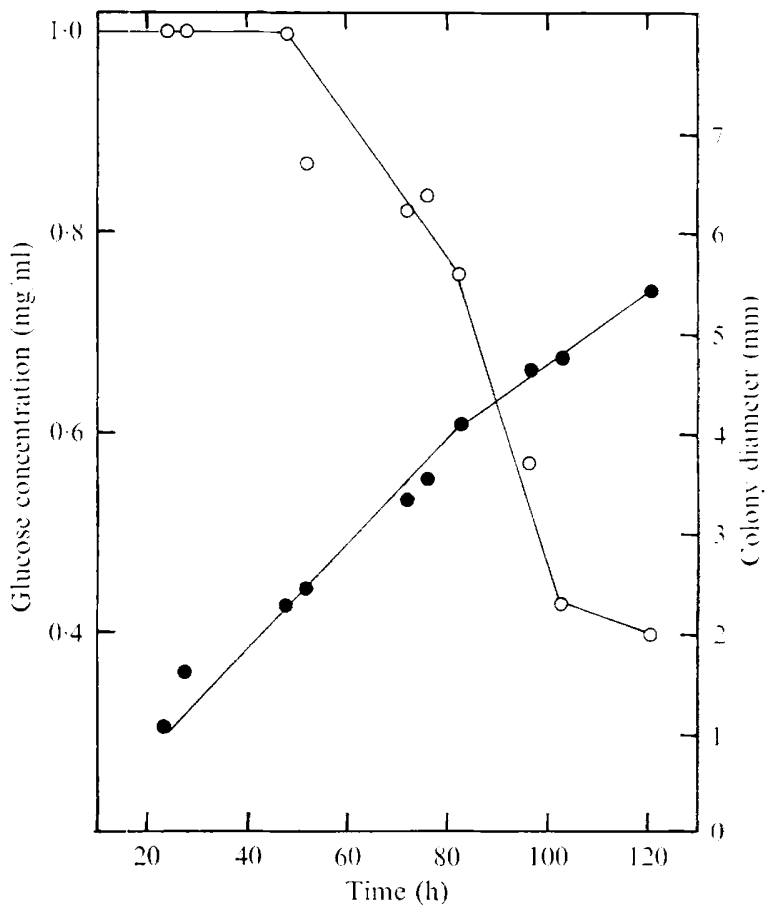

Fig. 3. A comparison of the glucose concentration at the edge of colonies and the colony diameter of Pseudomonas fluorescens incubated at $30^{\circ} \mathrm{C}$ on GSA with $0.1 \%$ glucose. $O$, Glucose concentration at colony edge;, average colony diameter.

points were observed, but at $0 \cdot 1 \%$ or below the $\Delta d / \Delta t$ decreased and transition points were observed (Table I). Earlier transition points were observed with lower concentrations of glucose.

Diffusion of glucose. By use of the methods of Lauffer (196I) and Shantz \& Lauffer (1962), duplicate coefficients of diffusion of glucose through GSA at $30^{\circ} \mathrm{C}$ were calculated to be 0.55 and $0.56 \times 10^{-5} \mathrm{~cm}^{2} / \mathrm{s}$. These values are in agreement with that of $0.60 \times 10^{-5} \mathrm{~cm}^{2} / \mathrm{s}$ for the free diffusion of glucose in water at $20^{\circ} \mathrm{C}$ (Washburn, 1929) and the findings of Stiles \& Adair (I92I), Stiles (I923) and Mann (I924) that the diffusion coefficients of salts in $2^{\circ}$ agar gels were 5 to $10 \%$ lower than those obtained by free diffusion in water.

Growth rate and glucose utilization by colonies on agar plates. The concentration of glucose in the medium at various distances from the edge of the colony was measured as described before. The data from one experiment is shown in Fig. 2. Each plot represents the concentration gradient of glucose from the colony edge to the body of the medium. The glucose concentration at the edge of the growing colony was obtained by extrapolation of the glucose concentration in the agar sections adjacent to the colony edge (shown by the dashed lines in Fig. 2). Probably this method gave too high values for the glucose concentration at the edge of the colonies and the glucose concentration gradient plots in Fig. 2 continued to curve downward. However, this extrapolation procedure appeared to be the only unbiased method of handling the available data. The relation between glucose concentration at the colony edge and colony diameter are presented in Fig. 3.

The total glucose available to the colony for growth was assumed to be the sum of the glucose diffusing through the peripheral boundary of the colony and the glucose made 
available by the increasing colony area. The amount of diffusible glucose was determined by Frick's First Law (Barrow, 196I):

$$
\mathrm{d} S / \mathrm{d} t=-D Q \mathrm{~d} c / \mathrm{d} t,
$$

where $\mathrm{d} S / \mathrm{d} t$ is the amount of glucose crossing the colony boundary in $\mathrm{mg} / \mathrm{s}$ and $Q$ is the cross-sectional area of the boundary in $\mathrm{cm}^{2} . Q$ was assumed to be the product of the circumference of the colony and the depth of the agar at the edge. At $97 \mathrm{~h}$ colony diameter was $0.464 \mathrm{~cm}$, the circumference $1.45 \mathrm{~cm}$, and agar depth $0.45 \mathrm{~cm}$. Therefore, at $97 \mathrm{~h}$, $Q=0.655 \mathrm{~cm}^{2} . D$ is the coefficient of diffusion of glucose through GSA and in our system equals $0.56 \times 10^{-5} \mathrm{~cm}^{2} / \mathrm{s}$. dc/dt, the concentration gradient, is the slope of the extrapolated initial linear portion of the plot in Fig. 2. At $97 \mathrm{~h}, \mathrm{~d} c / \mathrm{d} t=0.42 \mathrm{mg} / \mathrm{cm}-\mathrm{ml}$. Therefore

or

$$
\begin{aligned}
\mathrm{d} S / \mathrm{d} t & =\left(0.56 \times 10^{-5} \mathrm{~cm}^{2} / \mathrm{s}\right)\left(0.655 \mathrm{~cm}^{2}\right)(0.42 \mathrm{mg} / \mathrm{cm}-\mathrm{ml}) \\
& =0.165 \times 10^{-5} \mathrm{mg} / \mathrm{s}
\end{aligned}
$$

$$
D=0.59 \times 10^{-2} \mathrm{mg} / \mathrm{h} ;
$$

thus about $0.006 \mathrm{mg}$ of glucose was available to the colony from diffusion as it grew during the period from $96 \cdot 5$ to $97.5 \mathrm{~h}$.

Since the colony also grew during this period, it was necessary to determine the amount of glucose available from the medium already present under the area of new growth of the colony. It was assumed that the total amount of glucose in the segment which the colony covered in one hour was entirely available to the colony. Thus the amount of glucose available due to nutrients already present was determined from the product of segment volume and glucose concentration within the segment, e.g. at $97 \mathrm{~h} . \Delta d / \Delta t$ was $0.0035 \mathrm{~cm} / \mathrm{h}$, and thus the width of the segment was half this or $0.0017 \mathrm{~cm}$. Since this was a very thin section, its length was assumed to be constant and equal to the circumference of the colony which is $\mathrm{I} \cdot 45 \mathrm{~cm}$. The area of the segment is $0.0025 \mathrm{~cm}^{2}$ and its volume is $0.001 \mathrm{I} \mathrm{cm}$.

Since the glucose concentration at the colony edge at $97 \mathrm{~h}$ was $0.57 \mathrm{mg} / \mathrm{cm}^{2}$ (Fig. 3) the amount of glucose available to the colony from nutrients already present was the volume of the segment multiplied by the glucose concentration in the segment $(0.001 \mathrm{I} \mathrm{cm})\left(0.57 \mathrm{mg} / \mathrm{cm}^{2}\right)$, or $0.00063^{\circ} \mathrm{mg}$. The total available was then the sum of that available from diffusion and that available from existing nutrients, or, during the growth from 96.5 to $97.5 \mathrm{~h}$, it was $0.006+0.00063 \mathrm{mg}$ or $0.00663 \mathrm{mg}$. Similar calculations were made for the other times and these data are present in Table 2.

\section{DISCUSSION}

Fig. I is a numerical description of the fact that on spread plates those colonies in the more crowded portions of the plate are much smaller than those which are well separated. A single colony of Pseudomonas fluorescens on GSA continued to increase in diameter at a constant rate for 20 days, at which time observations were terminated. It was conjectured that this colony would have continued to grow at the same rate for as long as the nutrients were available or space was available in the Petri dish.

The abrupt decrease in the $\Delta d / \Delta t$ or transition point in crowded plates confirmed the decreases in the $\Delta d / \Delta t$ found by others (Shrewsbury, I93I ; Cruickshank, I934; Pirt, 1967), which Pirt (1967) speculated were due to a decreased availability of nutrients, although he showed that, at low glucose concentrations, the radial growth rate of colonies of Escherichia coli, Streptococcus faecalis and Klebsiella aerogenes was glucose-limited, a finding supported by our data for Pseudomonas fluorescens. 
Table I. The influence of glucose concentration in GSA on the $\Delta d / \Delta t$ and transition point of colonies of Pseudomonas fuorescens

* Not applicable.

$\begin{array}{cccc}\begin{array}{c}\text { Glucose concentration } \\ (\%, \mathrm{w} / \mathrm{v})\end{array} & \text { Transition point } & \text { Initial } & \text { Final } \\ \mathrm{I} & >\mathrm{h}) & 0.065 & \text { n.a.* } \\ 0.5 & >\mathrm{I} 60 & 0.063 & \text { n.a.* } \\ 0 . \mathrm{I} & 82 & 0.053 & 0.030 \\ 0.05 & 56 & 0.040 & 0.025 \\ 0.0 \mathrm{I} & 24 \dagger & 0.015 & 0.005\end{array}$

† Extrapolation of the initial linear portion of the diameter versus time plot indicated that for colonies of Pseudomonas fluorescens on GSA containing $0.01 \%$ glucose, the diameter increased more rapidly before the measurements were made.

Table 2. The amount of glucose available per hour to a colony of Pseudomonas fluorescens growing on GSA containing $0 \cdot \mathrm{I} \%$ glucose

$\begin{array}{cccccc}\begin{array}{c}\text { Time } \\ \text { (h) }\end{array} & \begin{array}{c}\text { Viable organism } \\ \text { count/colony }\end{array} & \begin{array}{l}\text { Due to } \\ \text { growth }\end{array} & \begin{array}{c}\text { Due to } \\ \text { diffusion }\end{array} & \text { Total } & \begin{array}{c}\text { Per viable organism } \\ \left(\times 10^{-11}\right)\end{array} \\ 24 & 15 \times 10^{6} & 0.0004 \mathrm{I} & - & 0.0004 \mathrm{I} & 2 \cdot 73 \\ 28 & 30 \times 10^{6} & 0.00059 & - & 0.00059 & 1 \cdot 96 \\ 48 & 127 \times 10^{6} & 0.00083 & - & 0.00083 & 0.65 \\ 52 & 142 \times 10^{6} & 0.00084 & 0.00097 & 0.00181 & 1 \cdot 27 \\ 72 & 44 \times 10^{7} & 0.00103 & 0.0018 & 0.0028 & 0.636 \\ 76 & 48 \times 10^{7} & 0.00109 & 0.0017 & 0.0028 & 0.58 \\ 83 & 120 \times 10^{7} & 0.00075 & 0.0030 & 0.0038 & 0.31 \\ 97 & 134 \times 10^{7} & 0.00063 & 0.0060 & 0.0066 & 0.49 \\ 103 & 128 \times 10^{7} & 0.00050 & 0.0074 & 0.0079 & 0.61 \\ 121 & 178 \times 10^{7} & 0.00052 & 0.013 & 0.0135 & 0.76\end{array}$

The amount of glucose appeared to control the time at which the transition point occurred (Table I); the transition point occurred earlier with lowered glucose concentrations. In Table 2, for a colony on $0.1 \%$ GSA the amount of glucose available to it per hour reached a maximum at $76 \mathrm{~h}$ and had started to decrease by the next reading at $83 \mathrm{~h}$, at which time the transition point and a rninimum value for the total amount of glucose available per viable organism in the colony were observed. Thus, the amount of glucose per viable organism in the colony controlled the growth of Pseudomonas fluorescens colonies. Despite this minimum for the amount of glucose per viable organism, the amount of glucose available to the colony from diffusion was constantly increasing.

After the transition point had occurred, although more total glucose was available to the colony, the colony had more organisms to utilize this glucose. In glucose-salts broth (GSB) with $0.1 \%$ glucose, Rieck ( 1963 ) found that $2.8 \times 10^{9}$ organisms $/ \mathrm{ml}$ utilized all the glucose with $36 \mathrm{~h}$ of growth. Thus, a colony of this number of organisms would be able to utilize much more glucose than would be available to it through growth and diffusion.

Of the three possible factors, physical crowding, accumulation of toxic metabolic products, and availability of nutrients, which could influence peripheral growth of a colony, availability of nutrients seemed to be the most important when physical crowding was absent. The accumulation of toxic metabolites should not be a factor with this strain of Pseudomonas fluorescens as Sinclair \& Stokes (1962) demonstrated during recycling studies that the re- 
growth of this strain in spent culture broth was solely nutrient dependent; when the broth was refortified with nutrients, maximum populations were obtained during seven cycles of growth.

Cruickshank (1934) reported that, with a luminous bacterium, lack of some diffusible nutrient, and not toxic metabolic products, were responsible for reducing the luminosity of colonies growing on crowded plates. During his study he removed portions of the agar close to the colonies and replaced them with either agar containing concentrated nutrients when luminosity reappeared, or with non-nutrient agar when it did not.

A linear increase of colony diameter with time suggested that only the organisms on the periphery of the colony were dividing (Palumbo et al. 1971). These peripheral bacteria may be the only organisms within the colony that were actively metabolizing. However, this peripheral ring of growth was of ever-increasing size and these bacteria alone could consume the glucose available to the colony. Further work should include data on nutrient transfer within the colony. This could be done by allowing nutrients to diffuse through a column of packed organisms in a manner similar to that done to study diffusion of glucose through GSA.

Pseudomonas fluorescens may not metabolize glucose to the same end products on GSA as in GSB. Recycling experiments with GSA, similar to those of Sinclair \& Stokes (1962) with GSB, might verify our conclusions about nutrient levels and growth of colonies, but they would be technically more difficult.

Palumbo (1963) attempted to measure the respiratory activities of single colonies of Pseudomonas fluorescens growing on trypticase soy agar (Baltimore Biological Laboratories) in Warburg flasks. Large amounts of oxygen were taken up by the uninoculated agar and no reliable data were obtained. Perhaps growth of colonies on cellophane might permit better measurement of the respiratory activity of colonies, and hence of their glucose consumption.

The data presented here support the general view that on $0.1 \%$ glucose GSA, colonies of Pseudomonas fluorescens increased linearly with time to a certain transition point after which time the colony diameters increased at a second and slower linear rate. This hypothesis is supported by the observation that the amount of glucose available per viable organism in the colony was a minimum at the transition point; and that there was an approximate linear increase in number up to $83 \mathrm{~h}$ and then an abrupt decrease in the rate of increase of viable cells when the colony viable organism count (Table 2) was plotted on log scale versus linear time. This abrupt decrease in the rate of viable organism increase correlated well with the colony diameter increases and the transition point. This apparent logarithmic increase of colony viable cells with time was in contrast to the non-logarithmic increase observed by Palumbo et al. (197I) and may be reflected in the fact that Palumbo et al. (197I) counted colonies from Petri dishes containing four or five colonies, while in this study, only colonies from Petri dishes with a single colony on them were counted.

We thank Theodore V. Kueper of Swift \& Company, Oakbrook, Illinois, U.S.A. for aid in the statistical analysis of the results obtained from colonies grown on high and low glucose concentrations. 


\section{REFERENCES}

Barrow, G. M. (1961). Physical Chemistry. New York: McGraw Hill.

BATES, P. K. (1929). Factors affecting the growth of surface colonies of bacteria. Ph.D. Thesis, Massachusetts Institute of Technology, Cambridge, Massachusetts, U.S.A.

Cooper, A. L., Dean, A. C. R. \& Hinshelwood, C. ( I968). Factors affecting the growth of bacterial colonies on agar plates. Proceeding of the Royal Society B I7I, I75-I99.

Cruickshank, J. (1934). A study of a luminous organism in relation to nutrition on agar. Journalof Pathology and Bacteriology 39, 141-148.

Johnson, M. G., Palumbo, S. A., Rieck, V. T. \& Witter, L. D. (1970). Influence of temperature on steadystate growth of colonies of Pseudomonas fluorescens. Journal of Bacteriology 103, 267-268.

LAUFFER, M. A. (1961). Theory of diffusion in gels. Biophysical Journal r, 205-213.

LEWIS, I. M. (1933). Secondary colonies of bacteria with special reference to Bacillus mycoides. Journal of Bacteriology 25, 359-387.

MANN, C. E. T. (1924). The determination of coefficients of diffusion in gels by means of chemical analysis, and a comparison of results obtained with those yielded by the indicator method. Proceedings of the Royal Society A ro5, 270--28I.

MonOD, J. (1942). Recherches sur la croissance des cultures bactériennes. Paris: Herman.

Palumbo, S. A. (1963). Growth parameters of surface colonies of bacteria. M.S. Thesis, University of Illinois, Urbana, Illinois, U.S.A.

Palumbo, S. A., Johnson, M. G., Rieck, V. T. \& Witter, L. D. (197I). Growth measurements on surface colonies of bacteria. Journal of General Microbiology 66, I 37-I 43.

PIRT, S. J. (1967). A kinetic study of the mode of growth of surface colonies of bacteria and fungi. Journal of General Microbiology 47, 18 I-197.

RIEck, V. T. (I963). The effect of glucose on surface colony growth of Pseudomonas fluorescens. M.S. Thesis, University of Illinois, Urbana, Illinois, U.S.A.

SHAH, K. K. \& IYER, V. N. (I96I). Secondary colony formation by Bacillus subtilis on Eosine Methylene Blue agar. Journal of Bacteriology 8I, 887-894.

Shantz, E. J. \& Lauffer, M. A. (1962). Diffusion measurements in agar gel. Biochemistry 1, 658-663.

Shrewsbury, J. F. D. ( I93I). Giant colony culture. Journal of Pathology and Bacteriology 34, 283-285.

Sinclair, N. A. \& Stokes, J. L. (I962). Factors which control maximal growth of bacteria. Journal of Bacteriology 83, I $147-$ I I 54 .

STILES, W. (1923). The indicator method for the determination of coefficients of diffusion in gels, with special reference to the diffusion of chlorides. Proceedings of the Royal Society A ro3, 260-275.

Stiles, W. \& Adair, G. S. (I92I). Penetration of electrolytes into gels. III. The influence of the concentration on the coefficient of diffusion of sodium chloride. Biochemical Journal 15, 620-628.

Washburn, E. W. (1929). International Critical Tables of Numerical Data, Physics, Chemistry, and Technology, vol. 5. New York: McGraw Hill. 